\title{
TOPOLOGICAL ENTROPY OF BLOCK MAPS
}

\author{
ETHAN M. COVEN
}

\begin{abstract}
We show that $h\left(f_{\infty}\right)=\log 2$ where $f_{\infty}$ is the map on the space of sequences of zeros and ones induced by the block map $f\left(x_{0}, \ldots, x_{k}\right)=x_{0}+$ $\Pi_{i=1}^{k}\left(x_{i}+b_{i}\right)$ where $k>2$ and the $k$-block $b_{1} \ldots b_{k}$ is aperiodic.
\end{abstract}

1. Introduction. Topological entropy, a conjugacy invariant of continuous selfmaps of compact Hausdorff spaces, was introduced in [AKM] in 1965. Over the years it has become an important concept in both topological and differentiable dynamics. For any excellent account of its place in present-day dynamics, see [B3].

Exact computations of topological entropy, other than for maps with zero entropy, appear to be rare. Exceptions are the Chebyshev polynomials [AM], endomorphisms of Lie groups [B2], Axiom A diffeomorphisms [B1], and a number of classes of subshifts. More common are results giving bounds for entropy, e.g., the results dealing with Shub's "entropy conjecture" [B3, Chapter 5], the recent results for maps of the interval [BF], [JR].

In this paper we will compute the topological entropy of a class of shift-commuting maps of the space $X$ of one-sided sequences of zeros and ones. In particular, we will prove the following.

TheOREM. Let $f\left(x_{0}, \ldots, x_{k}\right)=x_{0}+\prod_{i=1}^{k}\left(x_{i}+b_{i}\right)$, where $k \geqslant 2$ and the $k$-block $B=b_{1} \ldots b_{k}$ is aperiodic. Then $h\left(f_{\infty}\right)=\log 2$.

Here the arithmetic is to be done in $\operatorname{GF}(2), f_{\infty}: X \rightarrow X$ is defined by $\left[f_{\infty}(x)\right]_{i}=$ $f\left(x_{i}, \ldots, x_{i+k}\right)$ and $B$ is aperiodic means that there is no $p, 1 \leqslant p \leqslant k-1$, such that $b_{i}=b_{i+p}$ for $1 \leqslant i \leqslant k-p$.

It is rather surprising that, despite the finite nature of these maps [H, Theorem 3.4], there have been no previous entropy computations for shift-commuting maps.

2. Preliminaries. We assume the reader is familiar with the elementary properties of topological entropy, denoted $h()$.

Let $X$ denote the set of all sequences $x=x_{0} x_{1} x_{2} \ldots$ where each $x_{i}=0$ or 1 . Thus $X=\Pi_{0}^{\infty}\{0,1\}$. We give $\{0,1\}$ the discrete topology and $X$ the product topology. Then $X$ is a compact, metrizable space, homeomorphic to the Cantor set. A neighborhood base at $x \in X$ consists of all sets of the form $\left\{y \in X \mid y_{0} \ldots y_{n}=\right.$ $\left.x_{0} \ldots x_{n}\right\}$ where $n \geqslant 0$. 
An $n$-block is a concatenation of $n$ zeros and ones, i.e., a member of $\{0,1\}^{n}$. An $n$-block map is a function $f:\{0,1\}^{n} \rightarrow\{0,1\}$. Such a function also maps $(n+1)$ blocks to 2-blocks by

$$
f\left(x_{0}, \ldots, x_{n}\right)=\left(f\left(x_{0}, \ldots, x_{n-1}\right), f\left(x_{1}, \ldots, x_{n}\right)\right) .
$$

Similarly, $f$ maps $(n+2)$-blocks to 3-blocks, etc., and $f$ induces a continuous map $f_{\infty}: X \rightarrow X$ defined by $\left[f_{\infty}(x)\right]_{i}=f\left(x_{i}, \ldots, x_{i+n-1}\right)$. The shift $\sigma: X \rightarrow X$, defined by $[\sigma(x)]_{i}=x_{i+1}$, is induced by the 2-block map $s\left(x_{0}, x_{1}\right)=x_{1}$. It is well known that $h(\sigma)=\log 2$. The set of continuous, shift-commuting maps of $X$ to itself coincides with $\left\{f_{\infty} \mid f\right.$ is an $n$-block map, $\left.n \geqslant 1\right\}$ [H, Theorem 3.4].

For the purposes of evaluating block maps, it is convenient to think of the symbol set $\{0,1\}$ as $\operatorname{GF}(2)$, the field with two elements. The set of $n$-block maps coincides with the set of polynomials in $n$ variables over GF(2) of degree at most one in each variable [H, Theorem 19.1].

Composition of block maps is defined so that $(g \circ f)_{\infty}=g_{\infty} \circ f_{\infty}$. For example, if $f$ is a $(k+1)$-block map, then $f^{2}$ is the $(2 k+1)$-block map defined by

$$
f^{2}\left(x_{0}, \ldots, x_{2 k}\right)=f\left(f\left(x_{0}, \ldots, x_{k}\right), \ldots, f\left(x_{k}, \ldots, x_{2 k}\right)\right) .
$$

Let $f$ be a $(k+1)$-block map such that $f\left(x_{0}, \ldots, x_{k}\right)=g\left(x_{0}, \ldots, x_{k-1}\right)+x_{k}$ for some $k$-block map $g$. Then $f_{\infty}$ is conjugate to $\sigma^{k}$, the conjugacy being given by $[\psi(x)]_{n k+j}=f^{n}\left(x_{j}, \ldots, x_{n k+j}\right)$. Hence in this case $h\left(f_{\infty}\right)=k \log 2$. In fact, it can be shown that for any $(k+1)$-block map $f, h\left(f_{\infty}\right) \leqslant k \log 2$ with equality if and only if $f\left(x_{0}, \ldots, x_{k}\right)=g\left(x_{0}, \ldots, x_{k-1}\right)+x_{k}$ for some $k$-block map $g$.

3. The result. Let $f$ be a $(k+1)$-block map of the form

$$
f\left(x_{0}, \ldots, x_{k}\right)=x_{0}+\prod_{i=1}^{k}\left(x_{i}+b_{i}+1\right)
$$

where $k \geqslant 2$ and the $k$-block $B=b_{1} \ldots b_{k}$ is aperiodic, i.e., there is no $p$, $1 \leqslant p \leqslant k-1$, such that $b_{i}=b_{i+p}$ for $1 \leqslant i \leqslant k-p$. We will prove that $h\left(f_{\infty}\right)=$ $\log 2$.

These block maps were studied by the author and G. A. Hedlund in [CH] where they were used as feedback functions for nonlinear shift registers. The maps $f_{\infty}$ under consideration are continuous, finite-to-one [H, Theorem 5.5], map $X$ onto $X$ [H, Theorem 6.6] and commute with the shift. However they are not transitive, i.e., there is no point $x \in X$ with a dense $f_{\infty}$-orbit.

In the sequel, we will use juxtaposition of blocks to denote concatenation, omitting parentheses and commas. For example, we will write an expression such as $f(C D)=E$ when $C, D$ and $E$ are $k$-blocks.

The following notation from $[\mathbf{C H}]$ will prove helpful. Let $\tilde{0}=1, \tilde{1}=0$ and for an $n$-block $A=a_{1} \ldots a_{n}$ with $n \geqslant 2$, let $\tilde{A}=a_{1} \ldots a_{n-1} \tilde{a}_{n}$. Then by [CH, Lemma 6]

(i) $B$ is not an "interior block" for any of $B B, B \tilde{B}, \tilde{B} B$ or $\tilde{B} \tilde{B}$.

We collect together below some useful facts about the block map $f$. See [CH, Lemmas 7 and 9].

(ii) If $f(C D)=B$, then $C=B$ or $\tilde{B}$.

(iii) $f(C D)=\tilde{C}$ if and only if $D=B$. 
(iv) $f(C B)=\tilde{B}$ if and only if $C=B$.

(v) $f(C B)=B$ if and only if $C=\tilde{B}$.

(vi) If $B$ is not an interior block of $C D$, then $f(C D)=C$ or $\tilde{C}$.

Let $X_{B}=\left\{x \in X \mid\right.$ each $x_{i k} \ldots x_{i k+k-1}=B$ or $\left.\tilde{B}\right\}$, the set of concatenations of $B$ 's and $\tilde{B}$ 's. Then $X_{B}$ is closed and it follows from (i) and (vi) that $f_{\infty}$ maps $X_{B}$ to itself. Furthermore, $f_{\infty} \mid X_{B}$ is conjugate to $g_{\infty}$ where $g\left(x_{0}, x_{1}\right)=x_{0}+x_{1}$, the conjugacy being given by

$$
[\varphi(x)]_{i}= \begin{cases}1 & \text { if } x_{i k} \ldots x_{i k+k-1}=B \\ 0 & \text { if } x_{i k} \ldots x_{i k+k-1}=\tilde{B}\end{cases}
$$

Since $g_{\infty}$ is conjugate to the shift $\sigma$, it follows that $h\left(f_{\infty} \mid X_{B}\right)=\log 2$ and hence that $h\left(f_{\infty}\right) \geqslant \log 2$. We will show that $h\left(f_{\infty}\right) \leqslant \log 2$ by showing that for each $x \in X$, $h\left(f_{\infty} \mid \mathrm{cl} \theta(x)\right) \leqslant \log 2$, where $\theta(x)$ denotes the $f_{\infty}$-orbit of $x,\left\{f_{\infty}^{n}(x) \mid n=\right.$ $0,1, \ldots\}$. The result then follows from [G, Corollary 1].

Case 1. $x \in X_{B}$. Then $h\left(f_{\infty} \mid \operatorname{cl} \theta(x)\right) \leqslant h\left(f_{\infty} \mid X_{B}\right)=\log 2$.

Case 2. $B$ appears infinitely often in $x$ but $\sigma^{n}(x) \notin X_{B}$ for all $n \geqslant 0$. Write $x=A_{1} C_{1} A_{2} C_{2} \ldots$ using the following procedure. We illustrate the procedure for $B=011$ and $x=1001101001011011010101001101001100 \ldots$

Step 1. Underline the occurrences of $B$ in $x$.

$$
x=1001101001011011010101001101001100 \ldots
$$

Step 2. For each occurrence of $B$ in $x$, underline the maximal concatenation of $B$ 's and $\tilde{B}$ 's which ends in the indicated occurrence of $B$.

$$
x=10 \underline{011} 01001011011010101001101001100 \ldots
$$

Step 3. For each concatenation in Step 2 which is not a subconcatenation of another concatenation in Step 2, underline the maximal concatenation of $B$ 's and $\tilde{B}$ 's which can be obtained by extending to the right without overlapping the next concatenation.

$$
x=10 \underline{011010} 01011011010101001101001100 \ldots
$$

Step 4. Label the underlined concatenations of Step 3 by $C_{1}, C_{2}, \ldots$ and label the nonunderlined block preceding $C_{i}$ by $A_{i}$.

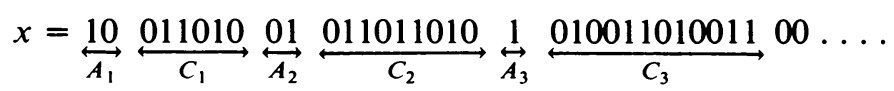

Note that in our example, $C_{1}=011010=B \tilde{B}$ is followed by $010=\tilde{B}$, but that this $\tilde{B}$ did not get underlined in Step 3 , for otherwise $C_{1}$ and $C_{2}$ would overlap.

The decomposition $x=A_{1} C_{1} A_{2} C_{2} \ldots$ has the following properties.

(1) $A_{i} \neq \varnothing$ if $i \geqslant 2$.

(2) $B$ does not appear in $A_{i}$.

(3) $A_{i}$ does not begin with $\tilde{B}$ if $i \geqslant 2$.

(4) $A_{i}$ does not end with $\tilde{B}$.

(5) $C_{i}$ is a concatenation of $B$ 's and $\tilde{B}$ 's.

Now write $f_{\infty}(x)=A_{1}^{1} C_{1}^{1} A_{2}^{1} C_{2}^{1} \ldots$ where $A_{i}^{1}$ has the same length as $A_{i}$ and $C_{i}{ }^{1}$ has the same length as $C_{i}$. In this case we say that " $A_{i}$ appears above $A_{i}^{1}$ ", etc. The 
meaning of the phrase " $D$ appears above $E$ " in similar situations will be clear from context.

Proposition. The decomposition $f_{\infty}(x)=A_{1}^{1} C_{1}^{1} A_{2}^{1} C_{2}^{1} \ldots$ also has properties (1)-(5).

Proof. Property (1) is clear.

(2) Suppose $B$ appears in $A_{i}^{1}$. Then by (iii), either $B$ or $\tilde{B}$ appears above $B$. Since $B$ does not appear in $A_{i}, \tilde{B}$ must appear above $B$. Then by (iii), the $k$-block in $x$ immediately following this appearance of $\tilde{B}$ is $B$. Thus $\tilde{B} B$ appears in $A_{i}$ or in $A_{i} C_{i}$. Since $B$ does not appear in $A_{i}$, this appearance of $B$ must be entirely in $C_{i}$. Then $A_{i}$ ends with $\tilde{B}$, contrary to (4).

(3) Let $i \geqslant 2$ and suppose $A_{i}^{1}$ begins with $\tilde{B}$. Let $D$ be the $k$-block in $x$ above this appearance of $\tilde{B}$ and let $E$ be the $k$-block in $x$ immediately following this appearance of $D$. Then $A_{i}$ begins with $D$ and so, by (2), $D \neq B$ and, by (3), $D \neq \tilde{B}$. Then by (iv), $B$ is an interior block of $D E$. This appearance of $B$ must be entirely in $C_{i}$ and hence $A_{i}$ and $C_{i}$ overlap. Thus $A_{i}^{1}$ does not begin with $\tilde{B}$.

(4) Suppose $A_{i}^{1}$ ends with $\tilde{B}$. Since $C_{i}$ begins with $B$ or $\tilde{B}$, by (iv) and (v), $A_{i}$ ends with $B$ or $\tilde{B}$, contrary to (2) or (4).

(5) Let $C_{i}=B_{1} \ldots B_{m}$ where each $B_{j}=B$ or $\tilde{B}$ and let $D$ be the initial $k$-block of $A_{i+1} C_{i+1}$. Then by (i) and (vi), $C_{i}^{1}=B_{1}^{1} \ldots B_{m-1}^{1} E$ where each $B_{j}^{1}=B$ or $\tilde{B}$ and $E=f\left(B_{m} D\right)$. But $B$ is not an interior block of $B_{m} D$, for otherwise $C_{i}$ and $C_{i+1}$ would overlap. Then by (vi), $E=B_{m}$ or $\tilde{B}_{m}$, i.e., $E=B$ or $\tilde{B}$.

Since the proof of the proposition involved only the properties of the original decomposition and not the procedure used to obtain them, it follows that for each $n \geqslant 1$, the decomposition $f_{\infty}^{n}(x)=A_{1}^{n} C_{1}^{n} A_{2}^{n} C_{2}^{n} \ldots$, where $A_{i}^{n}$ has the same length as $A_{i}$ and $C_{i}^{n}$ has the same length as $C_{i}$, also has properties (1)-(5).

Define $A_{i}^{1}=A_{i}$ and $C_{i}^{0}=C_{i}$. Let $i$ be fixed and consider the sequences of blocks $\left\{A_{i}^{0}, A_{i}^{1}, \ldots\right\}$ and $\left\{C_{i}^{0}, C_{i}^{1}, \ldots\right\}$.

Let $D^{n}$ be the terminal $k$-block of $C_{i}^{n}$ and let $E^{n}$ be the initial $k$-block of $A_{i+1}^{n} C_{i+1}^{n}$. Then $B$ is not an interior block of $D^{n} E^{n}$, so by (vi), $D^{n+1}=D^{n}$ or $\tilde{D}^{n}$. By (2), $E^{n} \neq B$, so by (iii), $D^{n+1}=D^{n}$. Thus the terminal $k$-block of $C_{i}^{n}$ is the same for all $n$ and therefore the sequence $\left\{C_{i}^{0}, C_{i}^{1}, \ldots\right\}$ is periodic, say with period $q_{i}$.

Let $F^{n}$ be the initial $k$-block of $C_{i}^{n}$. Since $B$ does not appear in any $A_{i}^{n}$ and $B$ is not an interior block of $A_{i}^{n} F^{n}$, it follows from (vi) and (iii) that $A_{i}^{n+1}=A_{i}^{n}$ or $\widetilde{A_{i}^{n}}$. Therefore the sequence $\left\{A_{i}^{0}, A_{i}^{1}, \ldots\right\}$ is periodic, with (not necessarily least) period $p_{i}=2 q_{i}$.

Define $D_{i}^{n}=A_{i}^{n} C_{i}^{n}$. Then the sequence of blocks $\left\{D_{i}^{0}, D_{i}^{1}, \ldots\right\}$ is periodic with period $p_{i}$. Since $f_{\infty}^{n}(x)=D_{1}^{n} D_{2}^{n} \ldots$ and $D_{i}^{n}$ appears above $D_{i}^{n+1}$, it follows that $f_{\infty} \mid \operatorname{cl} \Theta(x)$ is conjugate to a rotation on the compact group $\mathbf{Z}_{p_{1}} \times \mathbf{Z}_{p_{2}} \times \cdots$ and hence $h\left(f_{\infty} \mid \mathrm{cl} \theta(x)\right)=0$.

Case 3. $x \notin X_{B}$ but $\sigma^{n}(x) \in X_{B}$ for some $n \geqslant 1$. Then, in a manner similar to Case $2, f_{\infty} \mid \operatorname{cl} \theta(x)$ is conjugate to the product of a rotation on a finite group and $f_{\infty} \mid \operatorname{cl} \vartheta(y)$ for some $y \in X_{B}$. Hence $h\left(f_{\infty} \mid \operatorname{cl} \vartheta(x)\right)=h\left(f_{\infty} \mid \operatorname{cl} \vartheta(y)\right) \leqslant \log 2$. 
Case 4. $B$ appears only finitely often in $x$. Then $f_{\infty} \mid \mathrm{cl} \theta(x)$ is conjugate to a rotation on a finite group and hence $h\left(f_{\infty} \mid \mathrm{cl} \vartheta(x)\right)=0$.

Finally, by [G, Corollary 1], $h\left(f_{\infty}\right)=\sup _{x \in X} h\left(f_{\infty} \mid \operatorname{cl} \Theta(x)\right) \leqslant \log 2$, so $h\left(f_{\infty}\right)=$ $\log 2$.

\section{BIBLIOGRAPHY}

[AKM] R. L. Adler, A. G. Konheim and M. H. McAndrew, Topological entropy, Trans. Amer. Math. Soc. 114 (1965), 309-313. MR 30 \#5291.

[AM] R. L. Adler and M. H. McAndrew, The entropy of Chebyshev polynomials, Trans. Amer. Math. Soc. 121 (1966), 236-241. MR 32 \#6432.

[B1] R. Bowen, Markov partitions for Axiom A diffeomorphisms, Amer. J. Math. 92 (1970), 725-747. MR 43 \#2740.

[B2] _ Entropy for group endomorphisms and homogeneous spaces, Trans. Amer. Math. Soc. 153 (1971), 401-414. MR 43 \#469.

[B3] __ On Axiom A diffeomorphisms, CBMS Regional Conf. Ser. in Math., no. 35, Amer. Math. Soc., Providence, R.I., 1978.

[BF] R. Bowen and J. Franks, The periodic points of maps of the disk and the interval, Topology 15 (1976), 337-342. MR 55 \#4283.

[CH] E. M. Coven and G. A. Hedlund, Periods of some nonlinear shift registers, J. Combinatorial Theory Ser. A 27 (1979), 186-197.

[G] T. N. T. Goodman, Relating topological entropy and measure entropy, Bull. London Math. Soc. 3 (1971), 176-180. MR 44 \#6934.

[H] G. A. Hedlund, Endomorphisms and automorphisms of the shift dynamical system, Math. Systems Theory 3 (1969), 320-375. MR 41 \#4510.

[JR] L. Jonker and D. Rand, A lower bound for the entropy of certain maps of the unit interval, University of Warwick, preprint, 1978.

Department of Mathematics, Wesleyan University, Middletown, Connecticut 06457 